\title{
Efectos de un programa de ejercicio neuromuscular en la condición física del adulto mayor de la comunidad: ensayo clínico aleatorizado en grupos paralelos y ciego doble
}

\author{
Effects of a neuromuscular exercise program on the physical condition \\ of the older adult in the community: randomized clinical trial in parallel \\ groups and double blind
}

*Aldo Martínez A., ${ }^{* *}$ Reinaldo Saez S., ${ }^{* * *}$ Pablo Troncoso G., ${ }^{* * * *}$ Sebastián Astorga V., \& *****Guillermo Campos S.

Martínez, A., Saez, R., Troncoso, P., Astorga, S., \& Campos, G. (2021). Efectos de un programa de ejercicio neuromuscular en la condición física del adulto mayor de la comunidad: ensayo clínico aleatorizado en grupos paralelos y ciego doble. Revista Ciencias de la Actividad Física UCM, No 22(1), enero-junio, 1-12. DOI: http://doi.org/10.29035/rcaf.22.1.10

RESUMEN

Objetivo: Comparar los efectos de un programa de ejercicio neuromuscular (ENM) respecto a un programa de ejercicio municipal (EM) sobre la condición física en adultos mayores de la comunidad. Metodología: Ensayo clínico aleatorizado en grupos paralelos y ciego doble. Muestra constituida por 82 sujetos, edad X $=72$ años (DE = +/-5 años) pertenecientes a la Casa del Adulto Mayor de la ciudad de Talca. La selección fue de manera no probabilística por conveniencia, la asignación a los grupos mediante aleatorización estratificada y el ocultamiento de la asignación por medio de sobres cerrados. El cegamiento doble fue para los participantes y los evaluadores. Ambos grupos realizaron ejercicio en paralelo durante seis meses, tres veces a la semana. Las comparaciones inter-grupo de la condición física fueron analizados por medio de ANOVA multivariante de un factor. En todos los casos se adoptó un $p<0.05$ y se utilizó SPSS 25. Resultados: Los sujetos del grupo ENM lograron significativamente un mejor rendimiento en todas las variables de condición física respecto al grupo EM ( $p<0.05)$. Conclusión: El ENM mejoró significativamente la condición física de adultos mayores de la comunidad respecto a la aplicación de un programa de ejercicio municipal.

Palabras clave: Adulto mayor, ejercicio físico, esfuerzo físico.

\footnotetext{
* Dr. Ciencias de la Actividad Física, UCM, Chile, docente carrera de Kinesiología, miembro Grupo de Investigación en el Movimiento Humano (GIMH). http://orcid.org/0000-0002-8489-2557 | amartineza@uautonoma.cl

** Dro. Deporte y Actividad física, U. Iberoamericana de México, México, docente carrera de Kinesiología, miembro Grupo de Investigación en el Movimiento Humano (GIMH). https://orcid.org/0000-0002-0669-5056 | rsaezs@uautonoma.cl

*** Msc. Medicina y Ciencias del deporte, U. Mayor, Chile, docente carrera de Kinesiología, miembro Grupo de Investigación en el Movimiento Humano (GIMH). https://orcid.org/0000-0003-4320-0283 | ptroncosog@uautonoma.cl

**** Dr@. Ciencias del Deporte, U. Católica de Murcia, España, docente carrera de Kinesiología, miembro Grupo de Investigación en el Movimiento Humano (GIMH). https://orcid.org/0000-0002-8672-4987 | sastorgav@uautonoma.cl

***** Msc. TMO, U. Andrés Bello, Chile, docente carrera de Kinesiología, miembro Grupo de Investigación en el Movimiento Humano (GIMH). https://orcid.org/0000-0002-9492-2836 | guillermo.campos@uautonoma.cl
} 


\section{ABSTRACT}

Objective: To compare the effects of a neuromuscular exercise program (ENM) with respect to a municipal exercise program (EM) on the physical condition of older adults in the community. Methodology: Double blind, parallel group randomized clinical trial. Sample ma

de up of 82 subjects, age $X=72$ years (SD = +/- 5 years) belonging to the House for the Elderly in the city of Talca. Selection was non-probabilistic for convenience, group assignment by stratified randomization, and allocation concealment by sealed envelopes. Double blinding was for participants and assessors. Both groups exercised in parallel for 6 months, 3 times a week. The inter-group comparisons of physical condition were by means of one-way multivariate ANOVA. In all cases a p <0.05 was adopted and SPSS 25 was used. Results: The subjects of the ENM group achieved significantly better performance in all the physical condition variables compared to the EM group $(p<0.05)$. Conclusion: The ENM significantly improved the physical condition of older adults in the community with respect to the application of a municipal exercise program

Key words: Aged, Physical exercise, Physical Exertion.

\section{INTRODUCCIÓN}

América Latina se encuentra en un proceso de envejecimiento progresivo de su población; se estima que para el año 2050 existirán 180 millones de personas adultas mayores (AM) en la región (Leguizamón \& Kochmann, 2019).

En Chile, la efectividad de las políticas sociales implementadas desde la segunda mitad del siglo XX y las transformaciones demográficas, sociales, económicos y culturales experimentadas en las últimas tres décadas, han determinado un escenario social y cultural tal, que al comenzar el siglo XXI, el fenómeno de envejecimiento cobra mayor relevancia, debido principalmente al aumento de las expectativas de vida en la población (Miranda et al., 2020).

En este contexto, la población adulta mayor que vive en la comunidad deberá ser observada con más detención debido a la mayor probabilidad que presentan de sufrir una caída que provoca eventualmente la hospitalización o bien el ingreso a un centro de larga estadía (institucionalización) (Azcuy et al., 2020). Se estima que el 30\% de los AM de la comunidad sufrirán caídas a lo menos una vez a año, con la consecuente disminución de su condición física y, con ello, restricción de las actividades diarias, pérdida de independencia funcional y reducción en su interacción social. No obstante, es posible de prevenir esta serie de sucesos desfavorables en su calidad de vida, mediante la incorporación prematura a programas de actividad físicaregular (Muñoz \& Vargas, 2019).

La práctica de ejercicio físico regular es una de las principales estrategias no farmacológicas para envejecer de forma más saludable y mejorar la calidad de vida relacionada con la salud de las personas mayores (Charchabal et al., 2020). El envejecimiento activo puede llevar a la persona a incrementar su esperanza de vida y a disminuir los índices de morbilidad a lo largo de los años. Este fenómeno se debe a los efectos beneficiosos de la práctica de ejercicio físico sobre variables biológicas y psicosociales del ser y que se materializan de forma muy evidente en personas de avanzada edad (Tibán, 2020).

A grandes rasgos, de manera directa, el ejercicio físico mantiene y mejora la función músculo-esquelética, osteo-articular, cardiocirculatoria, respiratoria, endocrino-metabólica, inmunológica y psico-neurológica. De manera indirecta, la práctica de ejercicio físico tiene efectos beneficiosos en la mayoría, si no en todas, las funciones orgánicas del AM, contribuyendo a mejorar su funcionalidad, lo cual es sinónimo de mejor salud, mejor respuesta adaptativa y mayor 
resistencia ante las enfermedades (López, 2020). De hecho, realizar ejercicio físico de manera regular reduce el riesgo de desarrollar, o incluso morir, lo que hoy día son las principales y más graves causas de morbi-mortalidad en los países occidentales tales como accidentes vasculares e infarto al miocardio (Sánchez, 2020).

Sin embargo, todos estos beneficios serán dependientes de un adecuado diseño e intensidad de ejercicios adaptados a las características de la población mayor que, de no ser así, sus efectos podrían ser inferiores a lo esperado o bien iatrogénicos (Font et al., 2020). Tal es el caso de los programas de actividad física municipal dirigidos para adultos mayores de la comunidad, los que incluyen mayoritariamente estrategias de ejercicios aeróbicos, excluyéndose otras cualidades importantes como la fuerza, flexibilidad y estabilidad dinámica (Remache, 2019). Además, muchos de ellos requieren contar con elementos externos para su ejecución lo que, en algunas ocasiones, dificulta su implementación debido a falta de recursos (Savigne \& López, 2019).

En contraposición, los programas de ejercicios definidos como neuromusculares, frecuentemente utilizados en la recuperación funcional de deportistas y adultos jóvenes, se han destacado por su carácter funcional, integral, de bajo impacto osteoarticular y costo de implementación, ya que sólo utiliza el peso del cuerpo para su ejecución (Guzmán, et al., 2019). Éstos se fundamentan en el paradigma del péndulo invertido, lo que implica generar ambientes de trabajo inestables, los que estimularán sistemas aferentes, centrales y eferentes, desarrollando respuestas motoras y permitiendo incrementar el control motor del sujeto en beneficio de su condición física (Castellanos et al., 2020). No obstante, en base a la información recopilada, la presente modalidad de ejercicios aún no es incorporada en los diferentes programas de actividad física a nivel municipal.

En consecuencia, este estudio hipotetiza a partir de la mejora significativa de la condición física del adulto mayor de la comunidad producto de la aplicación de un programa de ejercicio neuromuscular respecto de un programa de ejercicio municipal. Por lo tanto, el objetivo del presente estudio fue comparar los efectos de ambos ejercicios sobre la condición física en adultos mayores de la comunidad.

\section{METODOLOGÍA}

\section{Tipo de estudio}

Se diseñó un estudio experimental tipo ensayo clínico aleatorizado (ECA) en grupos paralelos y ciego doble.

\section{Población, selección de la muestra,} tamaño muestral, técnica de

\section{aleatorización, técnica de ocultamiento de la asignación y cegamiento}

La población diana la constituyeron adultos mayores pertenecientes a la Casa del Adulto Mayor (CAM) de la ciudad de Talca con un total de 130 sujetos. La selección de la muestra fue de manera no probabilística por conveniencia con aquellos que cumplieron con los siguientes criterios de inclusión: capacidad cognitiva que les permitiera comprender órdenes, capacidad para desplazarse de manera independiente, menores de 85 años, no estar cursando episodio agudo de dolor musculoesquelético y no haber presentado accidente cerebrovascular en los últimos 6 meses. En cuanto a los criterios de exclusión, fueron considerados: sujetos mayores de 85 años, estar cursando episodio agudo de dolor musculoesquelético al momento de realizar la selección de la muestra y haber presentado accidente cerebrovascular en los últimos 6 meses. Respecto a los criterios de eliminación, fueron considerados: sufrir una caída durante la realización de los ejercicios, cursar episodio de dolor agudo musculoesquelético que le impida continuar con el programa y manifestar voluntariamente retirarse del estudio. Por lo tanto, el tamaño de la muestra quedó constituida por 82 AM, edad X = 72 años (DE 
$=+/-5$ años). De todos ellos, el 60\% fueron hombres y el $40 \%$ mujeres.

Fueron asignados 41 sujetos al grupo Ejercicio Municipal (grupo control) y 41 sujetos al grupo Ejercicio Neuromuscular (grupo experimental). La técnica utilizada de asignación fue mediante aleatorización estratificada, y la técnica de ocultamiento de la asignación fue por medio de sobres cerrados. Todos ellos tuvieron un seguimiento en paralelo y el 100\% de los sujetos finalizó el programa de ejercicios. El nivel de cegamiento del estudio fue considerado doble. Esto se explica porque los participantes desconocían qué tipo de ejercicios realizaron (ENM o EM), tal como los evaluadores, quienes también ignoraban la modalidad de actividad física que habían realizado los participantes (Cuadro 1).

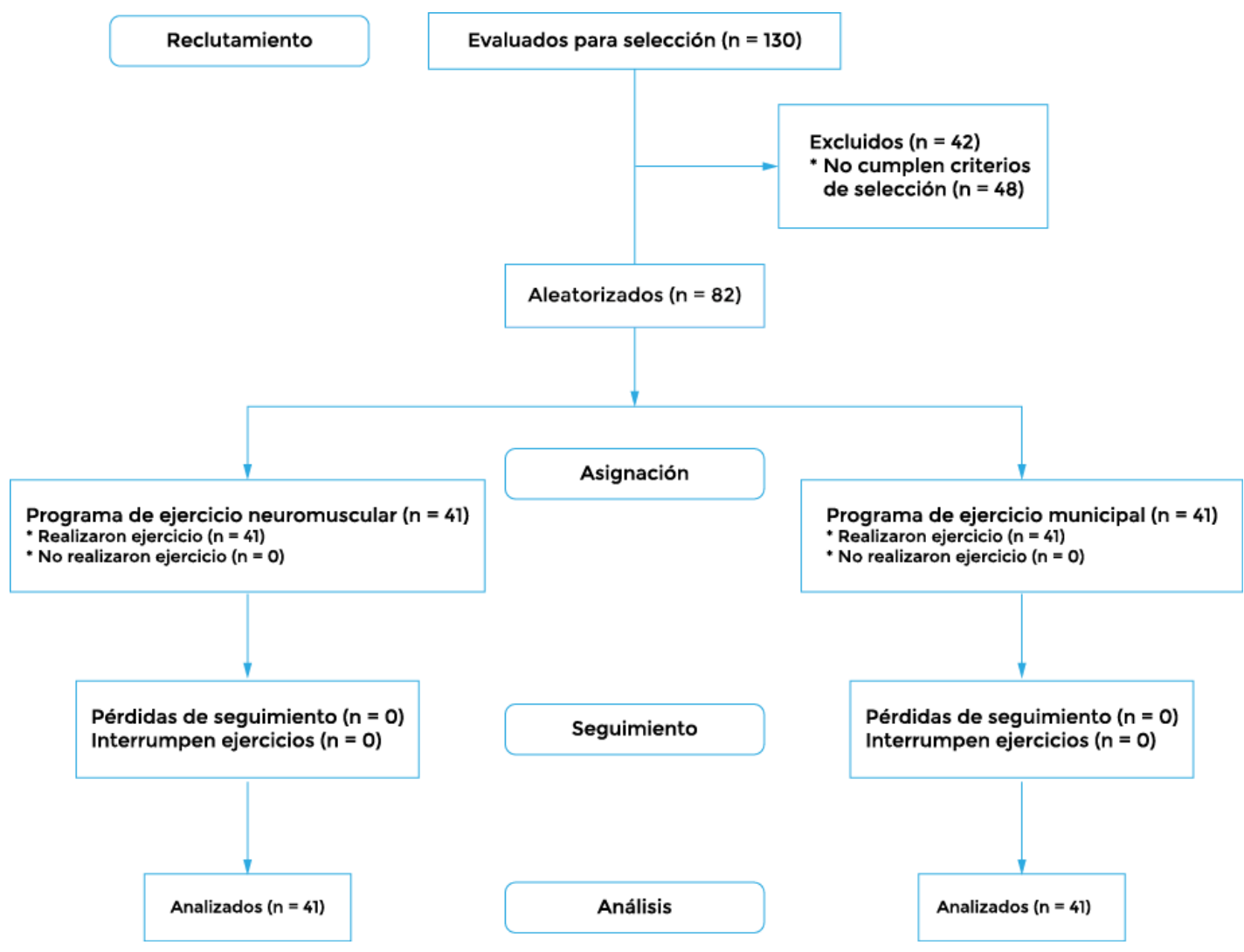

Cuadro 7. Diagrama de flujo CONSORT 2010.

\section{PROCEDIMIENTOS}

Se invitó a todos los sujetos a participar de la investigación con 30 días de anticipación. En dicha oportunidad, fueron informados sobre de su consentimiento a participar, el cronograma de actividades, las características de los distintos programas de ejercicios y el tipo de evaluaciones a realizar. Una vez reunida la muestra, se realizaron evaluaciones previas al inicio de los programas de ejercicios, las que consistieron en la recolección de datos personales, variables antropométricas, edad, presión arterial sistólica (PAS), presión arterial diastólica (PAD) y condición física. Todas ellas fueron realizadas en dos sesiones, con 3 días de intervalo, con el objetivo de verificar la confiabilidad de los instrumentos.

Las variables antropométricas de peso $(\mathrm{kg})$ y estatura (m) fueron medidas con ropa cómoda y descalzo. Se utilizó una báscula electrónica (United 
Kingdom, Ltd.) y un estadiómetro de aluminio (Seca Gmbh \& Co. KG, Hamburg, Germany). Se calculó el Índice de Masa Corporal utilizando la fórmula propuesta por Quetelet, donde [IMC = Peso $(\mathrm{kg}) /$ Estatura $(m)^{2}$. La presión arterial se evaluó mediante un esfigmomanómetro de mercurio y estetoscopio (Riester), se siguieron los procedimientos descritos por la Organización Panamericana de la Salud (OPS). Los valores se registraron después de permanecer al menos 10 minutos sentado (en reposo). Se registró la PAS y la PAD utilizando como referencia las fases I y $\vee$ de Korotkoff.

La condición física fue evaluada utilizando el Senior Fitness test. Para ello fueron consideradas las sub-áreas de fuerza muscular, flexibilidad, estabilidad dinámica y resistencia aeróbica.

La evaluación de la fuerza en extremidad superior consistió en que el AM debió estar sentado en unasilla con respaldo y utilizar una mancuerna de $2.27 \mathrm{~kg}$ (mujer) o $3.63 \mathrm{~kg}$ (hombre). Se registró el número de repeticiones de flexión el codo durante 30 segundos. Para la extremidad inferior, el AM sesentó en una silla conrespaldo y con los brazos extendidos en cruz. La medición consistió en contar el número de repeticiones que se levantó durante 30 segundos.

Para la evaluación de la flexibilidad de extremidad inferior, el AM se mantiene sentado en una silla, una pierna flexionada con el pie apoyado en el suelo y la otra, extendida. La medición consistió en flexionar el tronco con los brazos extendidos hacia adelante, intentando alcanzar los dedos de los pies o sobrepasarlos. En la extremidad superior, se tuvo que colocar de pie con su mano dominante sobre el mismo hombro y con la palma hacia abajo y los dedos extendidos. Desde esta posición dirigió su mano hacia la mitad de la espalda tan lejos como fuese posible. El otro brazo se colocó en la espalda rodeando la cintura con la palma de la mano hacia arriba intentando que se toquen los dedos medios de ambas manos. En ambas pruebas, la distancia entre los segmentos fue registrada con una cinta métrica marca Jonhson. Cuando no se alcanzó el contacto la distancia registrada fue considerada con signo negativo y cuando fue sobrepasado con signo positivo.

La estabilidad dinámica fue evaluada a través del tiempo que demoró el sujeto en levantarse de una silla, caminar hasta un cono situado a 2,44 m, girar y volver a sentarse. El tiempo fue registrado con un cronómetro Casio (1/100 seg).

Finalmente, la resistencia aeróbica fue calculada a través de la prueba de caminata de 6 minutos

El grupo experimental realizó un programa de ejercicio neuromuscular (ENM) y el grupo control un programa de ejercicio municipal (EM) (Cuadros 2 y 3). Ambos programas se aplicaron en paralelo durante 6 meses, 3 veces a la semana, con duración de 45 minutos. Finalizado el periodo de ejercicios, una semana después, se volvió a evaluar la condición física de los participantes.

\begin{tabular}{|c|c|c|}
\hline \multicolumn{3}{|c|}{$\begin{array}{c}\text { Protocolo ENM } \\
\text { Grupo experimental }\end{array}$} \\
\hline Calentamiento (15min) & ENM (25min) & Enfriamiento (5min) \\
\hline $\begin{array}{l}\text { Caminar por el entorno } \\
\text { Lateralización, rotación y } \\
\text { circunducción de cabeza y cuello } \\
\text { Inclinación de cuello } \\
\text { Flex-extensión, abducción-aducción } \\
\text { de brazos por encima de la cabeza. } \\
\text { Flexión de cadera y rodilla hasta } 90^{\circ} \text {. } \\
3 \text { repeticiones de } 1 \text { min de } \\
\text { estiramiento cabeza-cuello, } \\
\text { extremidades superiores e inferiores }\end{array}$ & $\begin{array}{l}20 \text { rep. } \times 3 \text { veces de elevación de talón. } \\
20 \text { rep. } \times 3 \text { veces de elevación de } \\
\text { antepié. } \\
3 \text { rep. de equilibrio unipodal hasta } 30 \\
\text { seg. } \\
3 \text { rep. de equilibrio con extremidades } \\
\text { inferiores cruzadas hasta } 30 \text { seg. }\end{array}$ & $\begin{array}{l}\text { Caminar a ritmo normal } \\
\text { durante } 5 \text { minutos. } \\
3 \text { repeticiones de } 1 \text { min de } \\
\text { estiramiento cabeza-cuello, } \\
\text { extremidades superiores e } \\
\text { inferiores. }\end{array}$ \\
\hline
\end{tabular}

Cuadro 2. ENM: Ejercicio Neuromuscular, rep: repeticiones, min: minutos, seg: segundos. 


\section{Protocolo EM}

\section{Grupo control}

\begin{tabular}{|c|c|c|}
\hline Calentamiento (15min) & EM (25min) & Enfriamiento (5min) \\
\hline $\begin{array}{l}\text { Caminar por el entorno } \\
\text { Lateralización, rotación y } \\
\text { circunducción de cabeza y cuello. } \\
\text { Inclinación de cuello. } \\
\text { Flex-extensión, abducción-aducción } \\
\text { de brazos por encima de la cabeza. } \\
\text { Flexión de cadera y rodilla hasta } 90^{\circ} \text {. } \\
3 \text { repeticiones de } 30 \text { seg de } \\
\text { estiramiento cabeza-cuello, } \\
\text { extremidades superiores e inferiores. }\end{array}$ & $\begin{array}{l}\text { Flexión y extensión de rodilla con } 1 \\
\text { kg de resistencia en cintura (sedente } \\
\text { sobre silla) } 15 \text { rep. } \times 3 \text { veces. } \\
\text { Flexión de rodilla utilizando respaldo } \\
\text { de una silla } 15 \text { rep. } \times 3 \text { veces. } \\
\text { Ejercicios de marcha, caminar } \\
\text { adelante en punta de pies, repetir } \\
\text { circuito de } 2.5 \mathrm{mt} \times 2 \text { veces }\end{array}$ & $\begin{array}{l}\text { Caminar a ritmo normal } \\
\text { durante } 5 \text { minutos } \\
3 \text { repeticiones de } 30 \text { seg de } \\
\text { estiramiento cabeza-cuello, } \\
\text { extremidades superiores e } \\
\text { inferiores. }\end{array}$ \\
\hline
\end{tabular}

Cuadro 3. EM: Ejercicio municipal, rep: repeticiones, res: resistencia, min: minutos, seg: segundos.

\section{Aspectos éticos del estudio}

Con el propósito de resguardar la integridad en la investigación, el presente estudio se adhirió a la declaración de Singapur y a los principios éticos de la asamblea de Helsinki. En virtud de aquello, fueron considerados los siguientes aspectos éticos: proveer de las condiciones ambientales óptimas para la ejecución segura de los programas de ejercicio, detener inmediatamente la sesión si el participante manifestara deseos de retirarse del entrenamiento, evaluar signos vitales antes, durante y después de cada sesión, realizar actividades de educación para el personal sanitario de todos los centros involucrados en la investigación y repetir el programa de ejercicio neuromuscular para los sujetos que conformaron el grupo control.

\section{Comité de ética}

El presente estudio fue aprobado con riesgo menor al mínimo, por el comité de ética científico de la Universidad Autónoma de Chile en acta Nº9-2019.

\section{Análisis estadísticos}

La normalidad de los datos fue verificada previamente por medio de la prueba de Kolmogorov-Smirnov, la comprobación de la confiabilidad del instrumento Senior Fitness Test se realizó por medio de $r$ de Pearson con 3 días de intervalo, las comparaciones antropométricas y fisiológicas intergrupo se calcularon a través de la prueba t para muestras independientes, la descripción de autopercepción en la condición física fue por medio de tablas de frecuencia, las comparaciones intra-grupo de condición física fue mediante prueba t para muestras relacionadas y las comparaciones inter-grupo de las variaciones en los distintos componentes de la condición física por medio de ANOVA multivariante de un factor. En todos los casos se adoptó un $\mathrm{p}<0.05$ y se utilizó SPSS 25.

\section{RESULTADOS}

Los datos tuvieron una distribución normal ( $p>0.05$ ), la confiabilidad del instrumento Senior Fitness test fue de $r=0.80$. En la tabla 1 se presentan las comparaciones antropométricas y fisiológicas intergrupo. Se puede observar que el grupo de EM presentó mayor peso e IMC en relación con el grupo de ENM ( $p<0.05)$. En el resto de los parámetros no hubo diferencias significativas $(p>0.05)$. 
Tabla 1

Características de la muestra estudiada.

\begin{tabular}{|c|c|c|c|c|}
\hline \multirow[t]{3}{*}{ Variables } & Grupos & $x$ & $\mathrm{DE}$ & \multirow[t]{3}{*}{$\mathrm{p}$} \\
\hline & $\mathrm{EM}(\mathrm{n}=41)$ & & & \\
\hline & ENM $(n=41)$ & & & \\
\hline \multirow{2}{*}{ Edad (años) } & EM & 71 & 5.5 & \multirow{2}{*}{ ns } \\
\hline & ENM & 73 & 6.3 & \\
\hline \multirow{2}{*}{ PAS (mmHg) } & EM & 130 & 11.9 & \multirow{2}{*}{ ns } \\
\hline & ENM & 130 & 9.5 & \\
\hline \multirow{2}{*}{ PAD $(\mathrm{mmHg})$} & EM & 75 & 7.5 & \multirow{2}{*}{ ns } \\
\hline & ENM & 76 & 7.0 & \\
\hline \multirow{2}{*}{ Peso (Kg) } & EM & 71 & 11.5 & \multirow{2}{*}{$0.04^{*}$} \\
\hline & ENM & 67 & 9.5 & \\
\hline \multirow{2}{*}{ Estatura (m) } & EM & 1.59 & 0.06 & \multirow{2}{*}{ ns } \\
\hline & ENM & 1.61 & 0.06 & \\
\hline \multirow{2}{*}{ IMC (kg/m2) } & EM & 27.7 & 4.7 & \multirow{2}{*}{$0.02^{*}$} \\
\hline & ENM & 25.9 & 2.9 & \\
\hline
\end{tabular}

EM: Ejercicio municipal, ENM: Ejercicio neuromuscular, PAS: Presión arterial sistólica, PAD: presión arterial diastólica, IMC: índice de masa corporal, ${ }^{*} p<0.05$, ns: no significativo.

La descripción de la autopercepción y condición física antes de iniciar los programas de ejercicios se presenta en la Tabla 2. Nótese que en ambos grupos el mayor porcentaje de sujetos la calificó como "aceptable" (EM=50\%, ENM=54\%). Esto indicaría una relativa homogeneidad en su autoevaluación inicial. Además, sólo el grupo de EM presentó la calificación de "muy mala". En la Tabla 3 se presentan los porcentajes de autopercepción de ambos grupos posterior a los programas de ejercicios. Se puede observar que ambas modalidades de ejercicios producen efectos beneficiosos en la autopercepción de condición física, alcanzando la calificación de "Buena" en el 73\% del grupo EM y $88 \%$ en el grupo ENM.

Tabla 2

Encuesta autopercepción de condición física pre-ejercicios.

\begin{tabular}{lcc}
\hline $\begin{array}{c}\text { Percepción } \\
\text { condición física }\end{array}$ & $\begin{array}{c}\text { Grupo } \\
\text { Control EM }\end{array}$ & $\begin{array}{c}\text { Grupo } \\
\text { Experimental ENM }\end{array}$ \\
\hline Muy mala & $2 \%$ & $0 \%$ \\
\hline Mala & $0 \%$ & $6 \%$ \\
\hline Aceptable & $50 \%$ & $54 \%$ \\
\hline Buena & $48 \%$ & $40 \%$ \\
\hline Muy buena & $0 \%$ & $0 \%$ \\
\hline Total & $100 \%$ & $100 \%$ \\
\hline$N$ & 41 & 41 \\
\hline
\end{tabular}

EM: Ejercicios municipales, ENM: Ejercicios neuromusculares, N: número de sujetos. 
Tabla 3

Encuesta de autopercepción de condición física post ejercicios.

\begin{tabular}{lcc}
\hline $\begin{array}{l}\text { Percepción } \\
\text { condición física }\end{array}$ & $\begin{array}{c}\text { Grupo } \\
\text { Control EM } \\
(\%)\end{array}$ & $\begin{array}{c}\text { Grupo } \\
\text { Experimental ENM } \\
(\%)\end{array}$ \\
\hline Muy mala & $0 \%$ & $0 \%$ \\
\hline Mala & $2 \%$ & $0 \%$ \\
\hline Aceptable & $25 \%$ & $12 \%$ \\
\hline Buena & $73 \%$ & $88 \%$ \\
\hline Muy buena & $0 \%$ & $0 \%$ \\
\hline Total & $100 \%$ & $100 \%$ \\
\hline$N$ & 41 & 41 \\
\hline
\end{tabular}

EM: Ejercicio municipal, ENM: Ejercicio neuromuscular, N: número de sujetos.

En la Tabla 4 se presentan las comparaciones intra-grupo de los valores alcanzados en cada una de las variables evaluadas para la condición física. Se puede observar que ambas modalidades tuvieron efectos significativos al cabo de 6 meses de entrenamiento $(p<$ 0.05). Esto demuestra que ambas estrategias son beneficiosas para mejorar la condición física del AM.

Tabla 4

Comparación intra-grupo variables de condición física pre-post ejercicio.

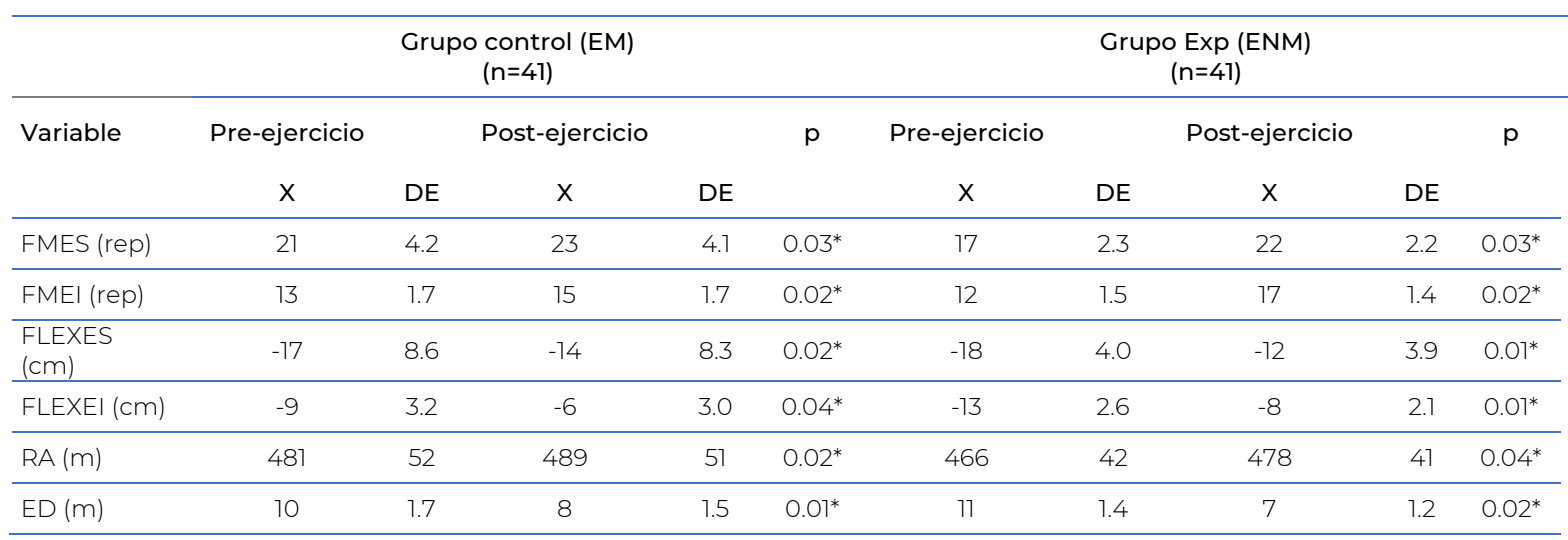

EM: Ejercicio municipal, ENM: ejercicio neuromuscular, X: promedio, DE: desviación estándar, FME: fuerza muscular ext superior, FMEI: fuerza muscular ext inferior, FLEXES: flexibilidad ext superior, FLEXEl: flexibilidad ext inferior, RA: resistencia aeróbica, ED: estabilidad dinámica, ${ }^{*} p<0.05$.

Las comparaciones inter-grupo de las variaciones alcanzadas en los distintos componentes de condición física se aprecian en la tabla 5. Obsérvese que los sujetos pertenecientes al grupo ENM lograron significativamente un mejor rendimiento en todas las variables respecto al grupo EM ( $p<0.05)$ especialmente en el aumento de la fuerza muscular en extremidades inferiores
$\left(R^{2}=0.76\right)$. En cambio, hubo una baja capacidad explicativa en el aumento de la estabilidad dinámica $\left(R^{2}=0.26\right)$. No obstante, la alta potencia del modelo corrobora la influencia del ejercicio ENM como variable independiente para provocar cambios significativos en la condición del sujeto (potencia = 1.00). 
Tabla 5

Comparación inter-grupo variaciones de condición física pre-post ejercicio.

\begin{tabular}{|c|c|c|c|c|c|c|c|}
\hline \multirow[t]{2}{*}{ Variable } & \multicolumn{2}{|c|}{$\begin{array}{l}\text { Grupo control (EM) } \\
(n=41)\end{array}$} & \multicolumn{2}{|c|}{$\begin{array}{c}\text { Grupo exp (ENM) } \\
(n=41)\end{array}$} & \multirow[t]{2}{*}{$\mathrm{p}$} & \multirow[t]{2}{*}{$\mathrm{R}^{2}$} & \multirow[t]{2}{*}{ Potencia } \\
\hline & $x$ & $\mathrm{DE}$ & $x$ & $\mathrm{DE}$ & & & \\
\hline VFMES (rep) & 1.93 & 0.72 & 4.32 & 1.01 & $0.03^{*}$ & 0.66 & 1.00 \\
\hline VFMEI (rep) & 1.93 & 0.72 & 4.90 & 0.97 & $0.02^{*}$ & 0.76 & 1.00 \\
\hline VFLXES $(\mathrm{cm})$ & 2.85 & 1.08 & 5.37 & 1.59 & $0.04^{*}$ & 0.47 & 1.00 \\
\hline VFLXEI (cm) & 2.88 & 1.07 & 5.39 & 1.51 & $0.01^{*}$ & 0.49 & 1.00 \\
\hline VRA (m) & 7.71 & 3.07 & 12.98 & 3.75 & $0.02^{*}$ & 0.38 & 1.00 \\
\hline VED (seg) & -2.27 & 1.30 & -3.95 & 1.58 & $0.04^{*}$ & 0.26 & 0.99 \\
\hline
\end{tabular}

EM: Ejercicio municipal, ENM: ejercicio neuromuscular, X: promedio, DE: desviación estándar, VFMES: variación fuerza muscular ext superior, VFMEl: variación fuerza muscular ext inferior, VFLXES: variación flexibilidad ext superior, VFLXEl: variación flexibilidad ext inferior, VRA: variación resistencia aeróbica, VED: variación estabilidad dinámica, ${ }^{*} \mathrm{p}<0.05$.

\section{DISCUSIÓN}

La presente investigación tuvo por objetivo comparar los efectos de la aplicación de un programa de ejercicio neuromuscular respecto a la aplicación de un programa municipal sobre la condición física en adultos mayores de la comunidad. Los resultados evidenciaron que el ENM mejoró significativamente todos los indicadores de condición física del AM descritos en la prueba Senior Fitness test. Este estudió demostró que el ENM mejoró la fuerza muscular de las extremidades superiores e inferiores en personan mayores de la comunidad sometidos a 6 meses de ejercicio. Esta afirmación ha sido descrita en otros estudios cuyos participantes fueron adultos jóvenes sanos (Guzmán et al., 2019). Al igual que en la presente investigación, quedó destacada su importancia para la mejoría en el desempeño funcional del sujeto (Donate et al., 2020).

Con relación a la flexibilidad, este estudio reportó que el ENM logró un aumento del 46\% en miembros superiores y un $47 \%$ en extremidades inferiores, respecto al EM. Esta información es concordante con otros estudios que aplicaron variadas estrategias de ejercicio y en todos ellos los mayores cambios se produjeron en extremidades inferiores (Carrera, 2019). En el presente estudio, estas diferencias pudieran explicarse por el mayor tiempo de elongación utilizado en el ENM, correspondiente a 1 minuto, respecto de los 30 segundos utilizados en el EM, pues la evidencia ha demostrado que a mayor tiempo de deformación de un tejido este adquirirá mayor viscoelasticidad (Herrera \& Zambrano, 2020).

En relación con la resistencia aeróbica, los resultados del presente estudio demostraron que el ENM incrementó en un 40\% la distancia recorrida, evidenciando un mayor efecto en el incremento de la capacidad cardiorrespiratoria respecto al EM. Estos resultados concuerdan con los de otros autores donde la aplicación de ejercicios funcionales incrementaron la distancia recorrida entre 10 y $25 \%$ (Peroni \& Goñi, 2020).

En cuanto a los resultados obtenidos para la estabilidad postural dinámica, este trabajo demostró queel ENM mejoró en un 42\% el equilibrio respecto al EM. Este beneficio fue reportado en estudios anteriores demostrando un efecto directo en la prevención de la caída y las subsecuentes fracturas asociadas (Contreras, 2019).

Finalmente, respecto a la autopercepción de condición física, hubo un mayor porcentaje de sujetos en el grupo ENM que la clasificaron como buena respecto al grupo de EM (88\% v/s 77\%). Esta diferencia puededeberse a los fundamentos teóricos del ENM, los que se basan en someter al sujeto a condiciones inestables a diferencia de los EM que se 
desarrollan en ambientes estables. Esto conlleva que, una vez alcanzado el dominio de los ejercicios, la percepción de condición física para enfrentar los desafíos de la vida diaria será mayor. Estosresultados fueron coincidentes a los encontrados en deportistas que fueron entrenados con ENM como estrategia de rehabilitación y reintegro deportivo (Huerta et al., 2019).

Este estudio presenta algunas potencialidades, como el alto porcentaje de sujetos que terminaron el programa de ENM (100\%) lo que indica su bajo impacto osteo-articular, el bajo costo de implementación y la diversidad de variables de condición física que lograron ser mejoradas. Estos hallazgos apoyan la incorporación de esta modalidad de ejercicio en las políticas públicas para la promoción y mantención por más tiempo de las capacidades funcionales del adulto mayor que vive

\section{REFERENCIAS BIBLIOGRÁFICAS}

Azcuy Aguilera, L., Camellón Pérez, A., \& Roque Doval, Y. (2020). Atención focalizada, desde la política social, a los adultos mayores institucionalizados del municipio Placetas, Cuba. Revista Novedades en Población, 16(31),

173-186.

http://www.novpob.uh.cu/index.php/NovP ob/article/view/435

Carrera Gualoto, D. (2019). Beneficios de la actividad física para mejorar la fuerza muscular, amplitud de movimiento articular $y$ flexibilidad en adulto mayor de 65-85 años del Centro Nuevo Renacer de la parroquia de Zámbiza durante el mes de septiembre a noviembre [Tesis de pregrado, Universidad Pontificia Católica del Ecuador]. http://repositorio.puce.edu.ec/handle/2200 o/16524 en la comunidad y, con ello, preservar su calidad de vida e interacción social.

\section{CONCLUSIÓN}

A partir delosresultadosobtenidos, se concluye queel ejercicio neuromuscular mejoró significativamente lacondición física y autopercepción de bienestar físico en adultos mayores de la comunidad respecto aquellos que lo hicieron con un programa municipal.

No obstante, variables como el tipo de alimentación, medicación y los niveles de calidad de vida no fueron evaluados. Se sugiere para nuevas investigaciones incorporar estas variables, las que permitirán analizar de manera más integral los efectos de esta modalidad de ejercicios en el adulto mayor de la comunidad.

Castellanos-Madrigal, S., Magdaleno-Navarro, E., Herrera-Rodríguez, V., García, M. D., \& Torres-Bugarín, O., \& (2020). Lesión del manguito rotador: diagnóstico, tratamiento y efecto de la facilitación neuromuscular propioceptiva. Residente, 15(1), 19-26. http://doi.org/10.35366/94039

Charchabal Pérez, D., Apolo Eguez, D., AlzolaTamayo, A., Espinoza Ordoñez, W. A., \& Yépez Herrera, E. (2020). Los potenciales efectos de la práctica regular de la actividad físicorecreativa en adultos mayores en la comunidad Época, Loja, Ecuador. Correo Científico Médico, 24(2), 685-700. http://www.revcocmed.sld.cu/index.php/co cmed/article/view/3639 
Contreras Alvarado, M. F. (2019). Factores Predictores de Recuperación Funcional a 3 meses en el Adulto Mayor hospitalizado por Fractura de Cadera [Tesis de especialidad, Instituto Tecnológico y de Estudios Superiores de Monterrey].

https://repositorio.tec.mx/handle/11285/636 236

Donate, F. P., Montiel, A, Merino, J. L. Chinchilla, \& A Castillo. (2020). La sarcopenia y la mejora de la capacidad funcional del adulto mayor. Innovación e investigación en actividad física y deporte para mayores. Servicio de Deportes. Diputación de Málaga. https://research.hanze.nl/ws/files/34968088 /INNOVACIO_N_E_INVESTIGACIO_N_EN_A CTIVIDAD_FI_SICA_Y_DEPORTE_PARA_MA YORES_compressed.pdf\#page=101

Font-Jutglà, C., Gimeno, E. M., Roig, J. B., da Silva, M. G., \& Villarroel, R. M. (2020). Efectos de la actividad física de intensidad suave sobre las condiciones físicas de los adultos mayores: revisión sistemática. Revista Española de Geriatría y Gerontología, 55(2), 98-106.

https://doi.org/10.1016/j.regg.2019.10.007

Guzmán-Muñoz, E., Daigre-Prieto, M., SotoSantander, K., Concha-Cisternas, Y., Méndez-Rebolledo, G., Sazo-Rodríguez, S., \& Valdés-Badilla, P. (2019). Efectos de un entrenamiento neuromuscular sobre el control postural de voleibolistas universitarios con inestabilidad funcional de tobillo: estudio piloto. Archivos de Medicina del Deporte, 36(5), 283-287. https://archivosdemedicinadeldeporte.co m/articulos/upload/orO2_guzman.pdf
Herrera Valencia, M. C., \& Zambrano Fuel, M. S. (2020). Efectividad de la elongación activa en isquiosurales para mejorar la flexibilidad en bailarinas y futbolistas profesionales: revisión sistemática. [Tesis de pregrado, Universidad de la Américas]. http://dspace.udla.edu.ec/jspui/handle/330 00/12895

Huerta Ojeda, Á. C., Casanova Sandoval, D. A., \& Barahona-Fuentes, G. D. (2019). Métodos de entrenamiento propioceptivos como herramienta preventiva de lesiones en futbolistas: una revisión sistemática. Archivos de Medicina del Deporte, 36(3), 173-180.

https://archivosdemedicinadeldeporte.co m/articulos/upload/revi_huerta.pdf

Leguizamón, M. A., \& Kochmann, S. (2019). El envejecimiento en América Latina y el Caribe: las dos posturas. Cómo estamos en Paraguay. Revista Científica Estudios e Investigaciones, $\quad 8(1), \quad$ 101-108. https://doi.org/10.26885/rcei.8.1.101

López Vidal, F. J. (2020). Presencia y características de la actividad física en centros de mayores en Sevilla [Tesis de doctorado, Universidad Pablo de Olavide]. https://rio.upo.es/xmlui/handle/10433/8636

Miranda Muñoz, N., Valenzuela Cofré, C., \& Vilches Gutierrez, M. (2020). Revisión sistemática de políticas sociales orientadas a personas mayores [Tesis de pregrado, Universidad Católica de la Santísima Concepción]. http://repositoriodigital.ucsc.cl/handle/2502 2009/2107

Muñoz Martínez, L. Y., \& Vargas Arévalo, M. (2019). Funcionalidad y calidad de vida del adulto mayor institucionalizado y no institucionalizado [Tesis de pregrado, Universidad Cooperativa de Colombia]. https://repository.ucc.edu.co/handle/20.500 $.12494 / 11194$ 
Peroni, B. B., \& Goñi, V. (2020). Efectos del entrenamiento aeróbico y de fuerza combinados vs entrenamiento aeróbico sobre capacidad aeróbica y fuerza muscular en pacientes con enfermedad de las arterias coronarias. Revista Ciencias de la Actividad Física UCM, 27(1), 1-17. https://doi.org/10.29035/rcaf.21.1.2

Remache, K. (2019). Ejercicios aeróbicos de bajo impacto como herramienta para combatir la depresión en los participantes del Proyecto de Desarrollo Integral Adulto Mayor "San Juan"[Tesis de maestría, Universidad Estatal de Bolívar]. http://dspace.ueb.edu.ec/bitstream/123456 789/3196/1/Tesis\%2OKlever\%2007-112019.pdf

Sánchez Aráuz, M. J. (2020). Estudio comparativo sobre la calidad de vida relacionada con la salud entre adultos mayores que realizan actividad física y sedentarios [Tesis de Pregrado, Universidad Pontificia Católica del Ecuador]. http://repositorio.puce.edu.ec/handle/2200 $\mathrm{o} / 18472$
Savigne Echemendía, J., \& López Pérez, Y. (2019). Ejercicios y actividades variadas para favorecer la incorporación del adulto mayor a la práctica masiva de ejercicios físicos. Revista Caribeña de Ciencias Sociales, 1-15. https://www.eumed.net/rev/caribe/2019/07/ incorporacion-adulto-ejercicios.html

Tibán Yánez, J. L. (2020). Capacidad funcional del adulto mayory su relación con la actividad física [Tesis de Pregrado, Universidad Técnica de Ambato]. https://repositorio.uta.edu.ec/jspui/handle/1 23456789/31370

\section{Dirección para correspondencia}

Doctor Aldo Martínez A.

Universidad Autónoma de Chile, sede Talca

Talca, Chile

Dirección Postal: 3460000

ORCID ID: http://orcid.org/0000-0002-8489-2557

Contacto:

amartineza@uautonoma.cl

Recibido: 15-12-2020

Aceptado: 14-06-2021 